\title{
Absent activation in medial prefrontal cortex and temporoparietal junction but not superior temporal sulcus during the perception of biological motion in schizophrenia: a functional MRI study
}

\author{
This article was published in the following Dove Press journal: \\ Neuropsychiatric Disease and Treatment \\ 17 November 2014 \\ Number of times this article has been viewed
}

\author{
Naoki Hashimoto ${ }^{1,2}$ \\ Atsuhito Toyomaki' \\ Masahiro $\mathrm{Hirai}^{3}$ \\ Tamaki Miyamoto' \\ Hisashi Narita' \\ Ryo Okubo' \\ Ichiro Kusumi' \\ 'Department of Psychiatry, \\ Graduate School of Medicine, \\ Hokkaido University, Sapporo, Japan; \\ ${ }^{2}$ Child and Adolescent Psychiatry, \\ Department of Psychiatry, University \\ of California, San Francisco, CA, USA; \\ ${ }^{3}$ Center for Development of Advanced \\ Medical Technology, Jichi Medical \\ University, Yakushiji, Shimotsuke, \\ Tochigi, Japan
}

Background: Patients with schizophrenia show disturbances in both visual perception and social cognition. Perception of biological motion (BM) is a higher-level visual process, and is known to be associated with social cognition. BM induces activation in the "social brain network", including the superior temporal sulcus (STS). Although deficits in the detection of BM and atypical activation in the STS have been reported in patients with schizophrenia, it remains unclear whether other nodes of the "social brain network" are also atypical in patients with schizophrenia.

Purpose: We aimed to explore whether brain regions other than STS were involved during $\mathrm{BM}$ perception in patients with schizophrenia, using functional magnetic resonance imaging (fMRI).

Methods and patients: Seventeen patients with schizophrenia, and 17 age- and sex- matched healthy controls, underwent fMRI scanning during a one-back visual task, containing three experimental conditions: (1) BM, (2) scrambled motion (SM), and (3) static condition. We used one-sample $t$-tests to examine neural responses selective to BM versus SM within each group, and two-sample $t$-tests to directly compare neural patterns to BM versus SM in schizophrenics versus controls.

Results: We found significant activation in the STS region when BM was contrasted with SM in both groups, with no significant difference between groups. On the contrary, significant activation in the medial prefrontal cortex (MPFC) and bilateral temporoparietal junction (TPJ) was found only in the control group. When we directly compared the two groups, the healthy controls showed significant greater activation in left MPFC and TPJ to BM versus SM than patients with schizophrenia.

Conclusion: Our findings suggest that patients with schizophrenia show normal activation to biologically and socially relevant motion stimuli in the STS, but atypical activation in other regions of the social brain network, specifically MPFC and TPJ. Moreover, these results were not due to atypical processing of motion, suggesting that patients with schizophrenia lack in the recruitment of neural circuits needed for the visual perception of social cognition.

Keywords: cognitive processing, social cognition, social brain network, mentalizing, theory of mind

\section{Introduction}

A large body of research indicates that patients with schizophrenia show deficits in low-level cognitive processing, such as contrast detection, to high-level cognitive processing, such as self-referential processing and affective recognition. ${ }^{1,2}$ One of the prominent symptoms in patients with schizophrenia is the impairment in social 
cognition, including the ability to infer intentions of other people [Theory of Mind (ToM) $]^{3,4}$ and perceive socially relevant cues such as detecting gaze direction and gestures (social perception)..$^{5}$ These deficits are evident not only in behavioral performance but in the neural patterns as well. Several neuroimaging studies in patients with schizophrenia have shown functional abnormalities in a network of brain areas thought to be considered the "social brain network", including right superior temporal sulcus (STS), left temporoparietal junction (TPJ), left medial prefrontal cortex (MPFC) $)^{6}$ and cingulate. ${ }^{5}$

In addition to these studies on the neural mechanisms underlying social cognition, visual perception has also been extensively studied in patients with schizophrenia. ${ }^{7,8}$ It is commonly known that deficient visual perception in this group is more prominent in the magnocellular pathway, which projects primarily to the dorsal visual stream and responds preferentially to low spatial frequency stimuli, low levels of luminance contrast, and motion. ${ }^{9}$ In fact, patients exhibit elevated contrast sensitivity thresholds, particularly to low spatial frequency stimuli, ${ }^{10}$ and longer backward masking effects when low luminance contrast was used. ${ }^{11}$ For studies on motion perception, patients have shown poorer velocity discrimination, ${ }^{12}$ global motion perception, ${ }^{13}$ and a deficit in the perception of biological motion (BM; a visual perceptual phenomenon whereby dozens of moving point-lights attached to joints of a human body is perceived as human action). ${ }^{14,15}$ Neural correlates of visual processing have also been investigated; researchers have found functional abnormalities in the vicinity of the motion-sensitive area (hMT+) in global motion perception and speed discrimination task, ${ }^{13}$ and in the STS during a BM perception task. ${ }^{15}$

Brittain et al studied the relationship between visual perception deficiency and social cognitive dysfunction using a battery of visual tasks (contrast sensitivity, visual masking, global motion, and BM), ${ }^{16}$ and showed that the schizophrenia group was significantly worse on all tasks. Among these four tasks, BM perception showed the most robust positive relationship with performance on a social perception task. Perceptual capacity for discerning BM also showed significant positive correlation with performance on the eye test, which is closely associated with ToM. ${ }^{17}$ Hence, converging evidence suggests that BM perception could be a hallmark of social cognition. ${ }^{18}$ Indeed, normal individuals can easily perceive not only vivid actions with only a dozen of pointlights attached to the major joints of a human body, ${ }^{19}$ but also sex, emotions, and other socially relevant information. ${ }^{20-22}$ Furthermore, several neuroimaging studies have reported that in addition to the STS region, other brain areas such as the premotor cortex, ${ }^{23}$ amygdala, ${ }^{24}$ and fusiform body area ${ }^{25}$ are involved in the perception of BM.

To the best of our knowledge, there are only two studies which focused on neural activation of patients with schizophrenia during BM perception, and neither of them mentioned neural activation in the social brain network other than STS. Kim et $\mathrm{al}^{15}$ showed abnormalities in the STS during a point-light BM task in schizophrenics using functional magnetic resonance imaging (fMRI) with an event-related task. They found no other abnormalities in the whole brain analysis, maybe because they used an event-related design. Because they showed normal STS function with blocked design task in eight out of ten of their schizophrenia patients, it is possible that we can find other activation in the social brain network using a blocked design. Thakkar et $\mathrm{al}^{26}$ implemented another fMRI study which examined brain activation during imitative versus nonimitative actions and observation of moving hands versus a moving spatial cue. In this study, they found abnormalities in the mirror neuron system in the patient group, but not in the social brain network, possibly because they adapted the tasks which had been originally used in the studies of the mirror neuron system.

In this study, we aimed to explore whether other parts of the social brain network other than the STS are involved in BM perception in patients with schizophrenia. We scanned 17 patients with schizophrenia and 17 healthy comparison subjects in a blocked design task with BM animations comprised of point-light motion stimuli performing various social activities, including emotional gestures and pretended actions to generate a robust blood oxygenated level-dependent (BOLD) signal in the social brain network. Since prior studies have suggested that patients with schizophrenia have difficulty in ToM tasks supported by the social brain network, ${ }^{4,27}$ we hypothesized that activation patterns in the other parts of the network associated with socially relevant stimuli would be atypical during BM perception in patients with schizophrenia.

\section{Methods and patients Participants}

The Hokkaido University Hospital Ethical Committee approved all study procedures, and the study was conducted according to the principles expressed in the Declaration of Helsinki. Before beginning the study, a description of the study was given to all participants and written informed consent was obtained. 
Seventeen patients (eight females) who met the criteria for a Diagnostic and Statistical Manual of Mental Disorders, Fourth Edition, Text Revised (DSM-IV-TR) ${ }^{28}$ diagnosis of schizophrenia were recruited from Hokkaido University Hospital. Patients who had a history of head injury, seizure disorder, dementia, diabetes mellitus, or other significant laboratory results were excluded from the study. No patients had a history of substance misuse, including alcohol abuse. Subjects who were currently suicidal and women who were pregnant or breastfeeding were also excluded. Sixteen patients were taking second-generation antipsychotics, and one patient was taking a first-generation antipsychotic.

For the control group, seventeen subjects (four females) with no history of a DSM-IV-TR Axis I disorder were recruited from the community. None of the control patients had a neurological disorder or a first-degree relative with a $D S M-I V-T R$ Axis I disorder, nor were any of them receiving psychotropic medications.

The demographic data of the participants are shown in Table 1 . The severity of clinical symptoms was assessed by the Positive and Negative Syndrome Scale (PANSS), and social function was assessed by the Social and Occupational Functioning Assessment Scale (SOFAS) score by treating psychiatrists. There were no statistical differences in age or sex between these two groups. All subjects had normal or corrected-to-normal vision. Practice trials were performed before the experiment, and all the subjects were able to detect the motion of the dots.

\section{Stimuli}

Point-light motion stimuli were generated from motioncaptured stimuli obtained from the internet. ${ }^{29}$ In the initial stimulus, there were 26 point-lights; however, we subsequently reduced this number to 17 and used this 17 point-lights stimulus for all subjects. The point-lights were

Table I Demographic data

\begin{tabular}{llll}
\hline & Control & Schizophrenia & P-value \\
\hline $\mathrm{N} \mathrm{(m:f)}$ & $17(13: 4)$ & $17(9: 8)$ & $0.5^{\mathrm{b}}$ \\
Age $^{\mathrm{a}}$ & $27.7(4.3)$ & $311(6.2)$ & $0.10^{\mathrm{c}}$ \\
Duration of illness (years) $^{\mathrm{a}}$ & - & $7.1(6.1)$ & - \\
Amount of antipsychotics $^{\mathrm{a}}$ & - & $437.9(356.5)$ & - \\
(chlorpromazine equivalent, $\mathrm{mg})$ & & & \\
PANSS positive symptom $^{\mathrm{a}}$ & - & $15.2(5.3)$ & - \\
PANSS negative symptom $^{\mathrm{a}}$ & - & $20.3(6.0)$ & - \\
PANSS general symptom $^{\mathrm{a}}$ & - & $37.4(7.2)$ & - \\
SOFAS $^{\mathrm{a}}$ & - & $4 I(12.8)$ & - \\
\hline
\end{tabular}

Notes: ${ }^{2}$ Mean (standard deviation); ${ }^{\mathrm{b}} \chi^{2}$ test; ${ }^{\mathrm{c}}$ Mann-Whitney test. Abbreviations: $\mathrm{N}$, number; PANSS, positive and negative syndrome scale; SOFAS, social and occupational functioning assessment scale; $m$, male; $f$, female. attached to various positions of the body as follows (number of point-lights in parentheses): head (one), shoulders (two), chest (one), hips (three), elbows (two), wrists (two), thighs (two), knees (two), and ankles (two). The BM animations depicted 15 various activities, including running, jumping, throwing, and kicking. Furthermore, we specifically included several stimuli representing emotional gestures (eg, the "banzai" gesture, which represents a feeling of happiness) and pretended actions such as picking up an invisible object. For the scrambled motion (SM), the number of point-lights and their velocity vectors were identical to those in the BM stimulus, but the spatial locations of each point-light were randomized, as in previous studies. ${ }^{30-32}$ For the static (ST) condition, static images created by capturing the initial frame of the BM stimuli were presented. Visual stimuli were displayed using E-Prime (Psychological Software Tools, Pittsburgh, PA, USA). For all three kinds of animations and images, the joints were displayed as small, white dots against a black background. The stimulus image was $3^{\circ} \times 3^{\circ}$ in size, and visual stimuli were back-projected with a Digital Light Processing projector onto a screen located at the observer's feet. A periscope mirror attached to a birdcage head-coil was adjusted before the onset of the scans to maximize the viewing angle of the screen.

\section{Task and procedure}

All subjects underwent a blocked design. Each trial lasted 3 seconds and consisted of a visual stimulus (BM, SM, or ST) for 2 seconds with an interval of 1 second (Figure 1). Each block lasted 30 seconds and consisted of ten trials. Each session consisted of 12 seconds (four volumes) that were discarded prior to analysis to allow for MR stabilization followed by eight blocks. Every subject completed one scanning run which consisted of three experimental sessions for a total of 756 seconds. The images within the block were chosen pseudorandomly for each trial, with a $10 \%$ chance (one time in every block) of repetition in successive trials. The subjects were instructed to monitor the stimuli carefully and indicate sequential repetitions with a button press (oneback task). This instruction maintained the subject's level of attention throughout each block of trials.

\section{Image acquisition}

All brain images were collected using a 1.5 T GE Sigma scanner located within Hokkaido University, School of Medicine. All the subjects participated in scanning examinations that lasted approximately $30-40$ minutes. T1 anatomical images of the head (spin echo, repetition time [TR] $500 \mathrm{~ms}$, echo 


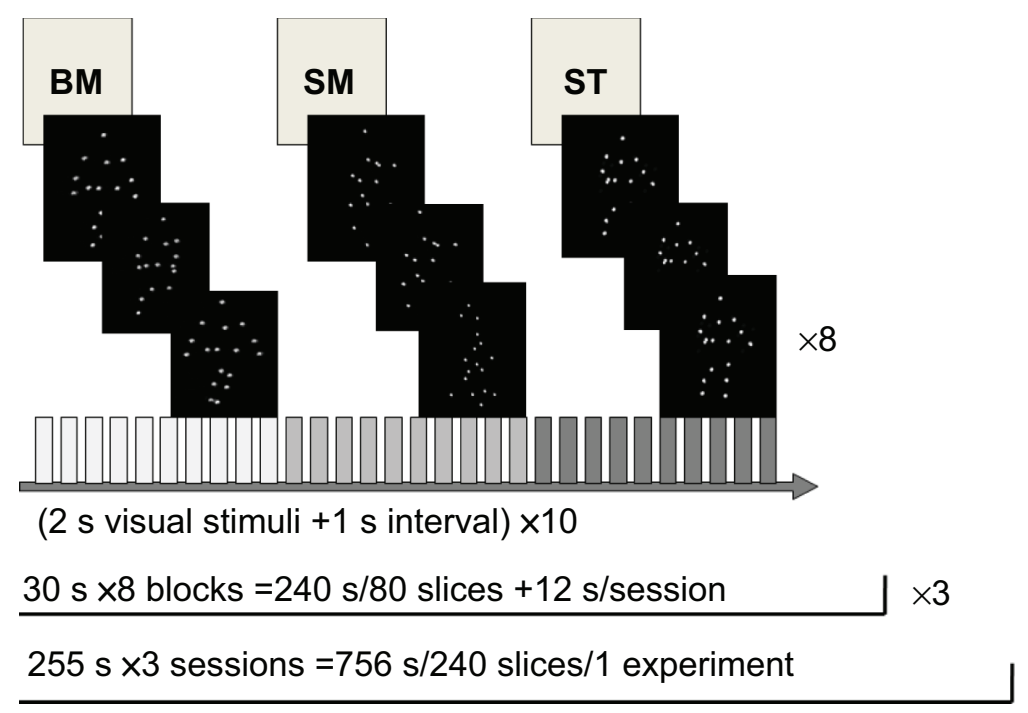

Figure I Task design.

Notes: Each block lasted 30 seconds and consisted of ten trials. Each trial consisted of a 2 second visual stimulus (BM, SM, or ST) and a I second interval (white cross). Each session consisted of 12 seconds that were discarded prior to analysis, followed by eight blocks. Each experiment involved three sessions.

Abbreviations: BM, biological motion; s, seconds; SM, scrambled motion; ST, static condition.

time [TE] $14 \mathrm{~ms}$, flip angle $90^{\circ}$, field of view $24 \mathrm{~cm} \times 24$ $\mathrm{cm}$, matrix size $256 \times 256$, slice thickness $4 \mathrm{~mm}$, interslice gap $1 \mathrm{~mm}, 20$ interleaved axial slices) and T2-star [T2*] functional images (gradient echo planar imaging [EPI], TR $3,000 \mathrm{~ms}$, TE $40 \mathrm{~ms}$, flip angle $90^{\circ}$, field of view $24 \mathrm{~cm}$ $\times 24 \mathrm{~cm}$, matrix size $64 \times 64$, slice thickness $4 \mathrm{~mm}$, interslice gap $1 \mathrm{~mm}, 20$ interleaved axial slices) were acquired during each of the examinations. The slice positions were chosen to cover almost the entire brain, including the entire occipital pole and the ventral surface of the temporal lobes.

\section{fMRI data analysis}

Data from the patient and control groups was analyzed using Statistical Parametric Mapping 8 software (SPM8; Wellcome Department of Cognitive Neurology, London, UK) implemented in MATLAB (Mathworks, Natick, MA, USA).

For each subject, the functional images from each run were realigned to the first scan, and normalized into the space defined by the International Consortium for Brain Mapping (ICBM) NIH P-20 project using the T1 template image $\left(\mathrm{ICBM}_{152}\right)$ supplied by SPM8. The data were smoothed using a Gaussian filter with the full-width-at-half-maximum parameters set to $8 \mathrm{~mm}$ at every dimension, and low frequency noise was removed by applying a high-pass filter (cut-off period, 128 seconds) to the fMRI time series at each voxel.

Significant hemodynamic changes for each condition were examined using a General Linear Model with box-car functions convolved with a hemodynamic response function. Statistical parametric maps of each contrast (BM-ST,
SM-ST, BM-SM) of the $t$-statistics were calculated on a voxel-by-voxel basis.

A fixed effect model was implemented for the group analysis. The contrast images were obtained from the singlesubject analyses and entered into the group analysis. At first, we compared the results from the BM-ST and SM-ST conditions in order to measure the activity during biological and SM, respectively, within each group using a one-sample $t$-test. In order to examine the effects of socially relevant perception, we assessed BM-SM (ie, BM perception without the effect of motion) within each group using a one-sample $t$-test. A statistical threshold of $\mathrm{q}$ (false discovery rate $[\mathrm{FDR}]$ ) $<0.05$ and an extended threshold of ten contiguous voxels were employed.

Finally, we made direct comparisons of these contrasts (BM-ST, SM-ST, BM-SM) between the normal and schizophrenia groups in order to assess the group difference for each effect, using two-sample $t$-tests. A statistical threshold of $P<0.0005$ (uncorrected) and an extended threshold of ten contiguous voxels were utilized.

\section{Results}

Mean performance on the one-back task was $86 \%$ for the normal control group and $81 \%$ for the schizophrenia group. No statistical difference was found between groups (Mann-Whitney $U$-test, $P=0.73$ ).

The neuroimaging results for both the groups are depicted in Figures 2-3. We first present the responses to BM versus ST and SM versus ST (Figure 2) before the main comparison of BM versus SM (Figure 3). 


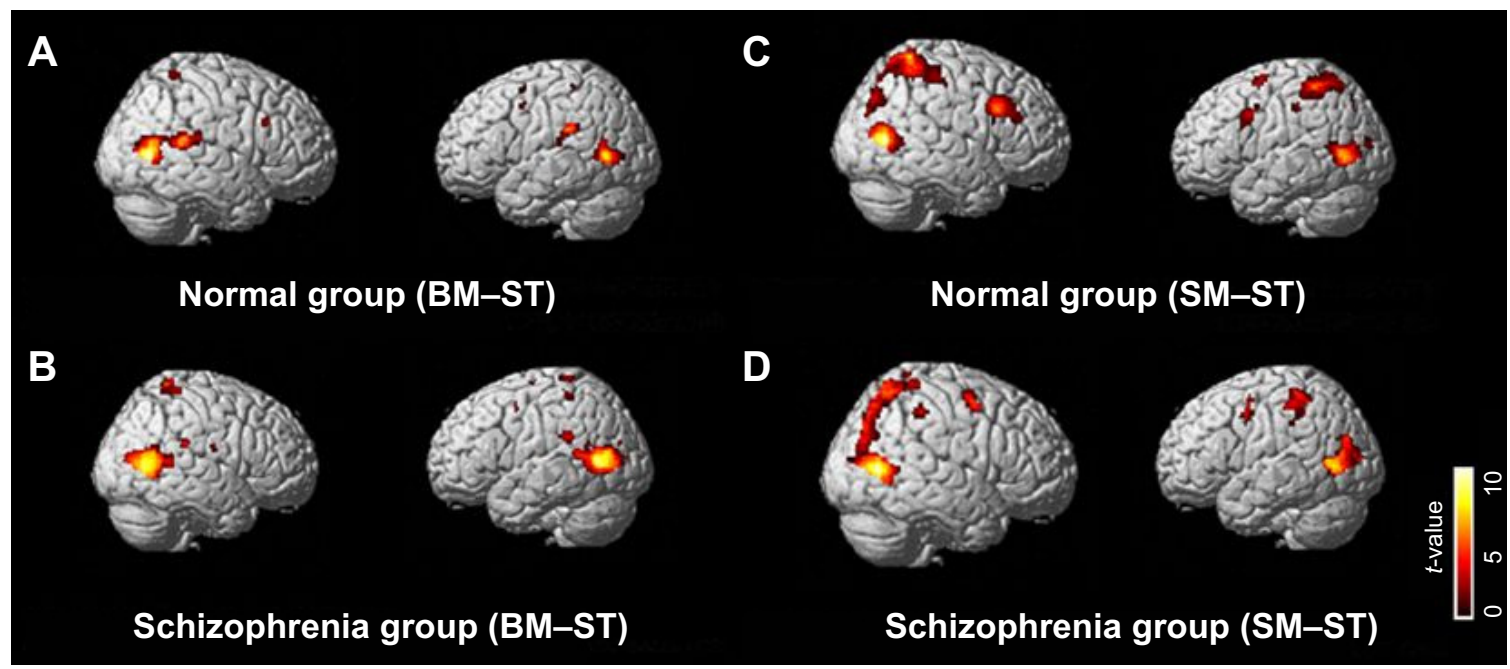

Figure 2 Brain regions significantly activated in the BM-ST (A, B) and SM-ST (C, D) conditions have been superimposed onto the SPM templates.

Notes: In both groups (normal and schizophrenia), the activated clusters covered the bilateral hMT+ and bilateral frontal regions in both the BM-ST and SM-ST conditions. Furthermore, in the SM-ST condition, the bilateral superior parietal lobules were activated in both the groups. A one-sample $t$-test, $q(F D R)<0.05$, and an extended threshold of ten contiguous voxels were employed. No significant activation was observed after subtraction of the results of schizophrenia group from those of normal control group in both the BM-ST and SM-ST conditions. A two-sample $t$-test, $P<0.0005$ (uncorrected) and an extended threshold of ten contiguous voxels were employed.

Abbreviations: BM, biological motion; FDR, false discovery rate; hMT+, motion-sensitive area; SM, scrambled motion; SPM, statistical parametric mapping; ST, static condition.

\section{Neural activation to biological motion and scrambled motion in normal controls and schizophrenics}

The comparisons of BM-ST and SM-ST observations yielded widespread brain activation, including frontal, temporal, parietal, and occipital regions, with the most robust activation shown in the bilateral temporal cortices in both the groups (normal group: Figure $2 \mathrm{~A}$ and $\mathrm{C}$, Table $2 \mathrm{~A}$, Table 3A; schizophrenia group: Figure 2B and D, Table 2B, Table 3B). In addition to these areas, we also found relatively

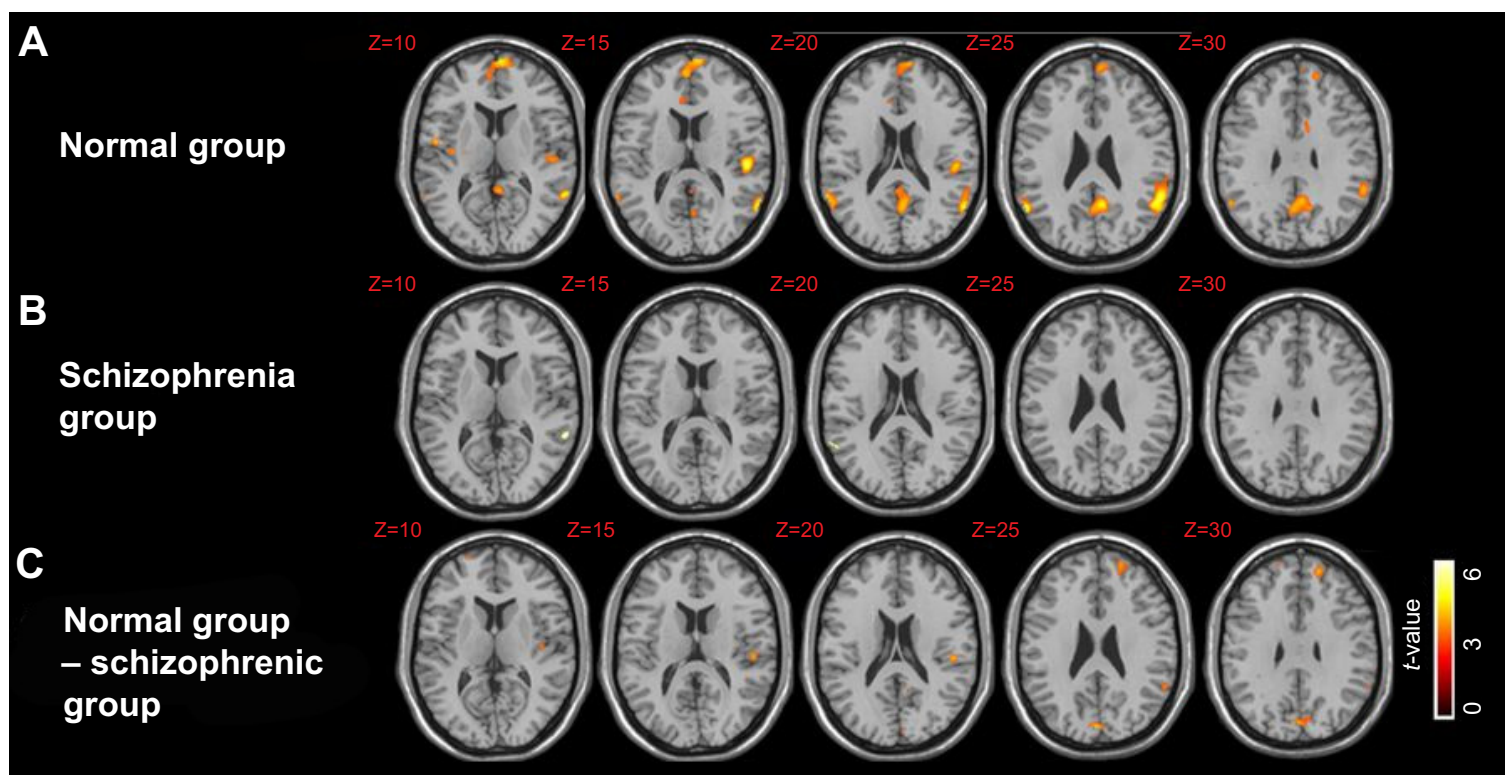

Figure 3 Brain regions significantly activated in the BM-SM conditions have been superimposed onto the SPM templates.

Notes: (A) Brain regions that were significantly activated in the normal group. The activated clusters covered the bilateral STS, bilateral medial frontal cortex, left precuneus, and middle cingulate. A one-sample $t$-test, $\mathrm{q}(\mathrm{FDR})<0.05$, and an extended threshold of ten contiguous voxels were employed. (B) Brain regions that were significantly activated in the schizophrenia group. The activated clusters included bilateral STS only. A one-sample $t$-test, $\mathrm{q}(\mathrm{FDR})<0.05$, and an extended threshold of ten contiguous voxels were employed. (C) Brain regions that were more activated in the normal group than in the schizophrenia group. The activated cluster included the left medial frontal gyrus, supramarginal gyrus, precuneus, cuneus, and middle cingulate. A two-sample $t$-test, $P<0.0005$ (uncorrected) and an extended threshold of ten contiguous voxels were employed. Z; z-value of each transverse slice.

Abbreviations: BM, biological motion; FDR, false discovery rate; hMT+, motion-sensitive area; SM, scrambled motion; SPM, statistical parametric mapping; STS, superior temporal sulcus. 
Table 2 Significantly activated clusters for the BM-ST contrast

\begin{tabular}{|c|c|c|c|c|c|c|c|}
\hline \multirow[t]{2}{*}{ Region } & & \multirow[t]{2}{*}{ BA } & \multicolumn{3}{|c|}{ MNI coordinates } & \multirow[t]{2}{*}{ Cluster size } & \multirow[t]{2}{*}{$t$-value } \\
\hline & & & $\mathbf{x}$ & $y$ & $\mathbf{z}$ & & \\
\hline \multicolumn{8}{|c|}{ A: normal group } \\
\hline \multicolumn{8}{|c|}{ Frontal lobe } \\
\hline $\mathrm{Lt}$ & Precentral gyrus & 6 & -44 & 2 & 46 & 12 & 4.41 \\
\hline $\mathrm{Lt}$ & Precentral gyrus & 6 & -40 & 2 & 58 & 10 & 4.03 \\
\hline $\mathrm{Rt}$ & Inferior frontal operculum & 48 & 44 & 20 & 30 & 23 & 4.00 \\
\hline \multicolumn{8}{|c|}{ Parietal lobe } \\
\hline $\mathrm{Rt}$ & Superior parietal lobule & 7 & 30 & -50 & 66 & 35 & 4.81 \\
\hline $\mathrm{Lt}$ & Postcentral gyrus & 3 & -30 & -38 & 60 & 24 & 3.97 \\
\hline \multicolumn{8}{|c|}{ Temporal lobe } \\
\hline Rt & Middle temporal lobe & 37 & 50 & -68 & 6 & 890 & 10.05 \\
\hline Lt & Middle temporal lobe & 37 & -56 & -66 & 6 & 404 & 5.96 \\
\hline $\mathrm{Lt}$ & Middle temporal lobe & 48 & -50 & -38 & 28 & 189 & 5.40 \\
\hline \multicolumn{8}{|c|}{ Occipital lobe } \\
\hline $\mathrm{Lt}$ & Lingual gyrus & II & -16 & 24 & -8 & 16 & 4.43 \\
\hline \multicolumn{8}{|c|}{ B: schizophrenia group } \\
\hline \multicolumn{8}{|c|}{ Frontal lobe } \\
\hline Lt & Precentral gyrus & 6 & -44 & 2 & 48 & 15 & 3.82 \\
\hline Rt & Precentral gyrus & 6 & 34 & 2 & 34 & 29 & 3.92 \\
\hline \multicolumn{8}{|c|}{ Parietal lobe } \\
\hline Rt & Precuneus & 5 & 10 & -50 & 70 & 149 & 4.54 \\
\hline Lt & Supramarginal gyrus & 48 & -54 & -42 & 26 & 79 & 4.42 \\
\hline $\mathrm{Lt}$ & Precuneus & 3 & -14 & -40 & 74 & 34 & 4.30 \\
\hline Rt & Precuneus & 7 & 26 & -56 & 32 & 19 & 4.13 \\
\hline Rt & Rolandic operculum & 48 & 54 & -10 & 20 & 10 & 3.91 \\
\hline Lt & Paracentral lobule & 6 & -100 & -12 & 70 & 20 & 3.88 \\
\hline Lt & Postcentral gyrus & 2 & -40 & -38 & 62 & 24 & 3.78 \\
\hline \multicolumn{8}{|c|}{ Temporal lobe } \\
\hline Rt & Middle temporal gyrus & 37 & 48 & -66 & 6 & 1,115 & 9.25 \\
\hline Lt & Middle temporal gyrus & 37 & -46 & -66 & 6 & 1,170 & 7.13 \\
\hline Lt & Inferior temporal gyrus & 36 & -36 & 0 & -30 & 48 & 4.04 \\
\hline Rt & Superior temporal gyrus & 48 & 54 & -34 & 22 & 51 & 3.80 \\
\hline \multicolumn{8}{|c|}{ Occipital lobe } \\
\hline $\mathrm{Lt}$ & Middle occipital lobe & 19 & -28 & -80 & 20 & 10 & 3.67 \\
\hline
\end{tabular}

Notes: The clusters showed significant activation for each condition. Each row shows the anatomical location of the activation and the corresponding BA, the MNI coordinates of the peak voxel, and the volume of activation. A one-sample $f$-test at a threshold of $q(F D R)<0.05$ and an extended threshold of ten contiguous voxels was employed. Abbreviations: BA, Brodmann's area; BM, biological motion; FDR, false discovery rate; Lt, left; MNI, Montreal Neurological Institute; Rt, right; ST, static condition.

large BOLD responses in the bilateral superior parietal lobule (SPL) in the normal group and right SPL in the schizophrenia group in SM-ST contrast. However, the schizophrenic and normal control groups did not significantly differ in brain activation to BM-ST and SM-ST conditions [two-sample $t$-test, $P<0.0005$ (uncorrected) and an extended threshold of ten contiguous voxels].

\section{Neural activation to socially relevant stimuli in normal controls and schizophrenics}

In the control group only, bilateral STS, bilateral MPFC, precuneus, and middle cingulate responded significantly more to BM than SM stimuli (Table 4A, Figure 3A). In the schizophrenic group, only bilateral STS responded significantly more to BM than SM stimuli (Table 4B, Figure 3B). Finally, we directly compared the two groups and found significantly increased neural activation in the left MPFC, TPJ (supramarginal gyrus), precuneus, cuneus, and middle cingulate [two-sample $t$-test, $P<0.0005$ (uncorrected) and an extended threshold of ten contiguous voxels] in the control group compared to the schizophrenic group (Table 4C, Figure 3C).

\section{Discussion}

In the current experiment, we found enhanced neural activation to socially relevant stimuli in the left MPFC and TPJ for normal controls compared to patients with schizophrenia. Consistent with previous neuroimaging studies on BM 
Table 3 Significantly activated clusters for the SM-ST contrast

\begin{tabular}{|c|c|c|c|c|c|c|c|}
\hline \multirow[t]{2}{*}{$\overline{\text { Region }}$} & & \multirow[t]{2}{*}{ BA } & \multicolumn{3}{|c|}{ MNI coordinates } & \multirow[t]{2}{*}{ Cluster size } & \multirow[t]{2}{*}{$t$-value } \\
\hline & & & $\mathbf{x}$ & $y$ & $\mathbf{z}$ & & \\
\hline \multicolumn{8}{|c|}{ A: normal group } \\
\hline \multicolumn{8}{|c|}{ Frontal lobe } \\
\hline Rt & Inferior frontal operculum & 44 & 54 & 20 & 32 & 424 & 5.95 \\
\hline Lt & Precentral gyrus & 6 & -32 & 2 & 62 & 91 & 4.77 \\
\hline $\mathrm{Lt}$ & Precentral gyrus & 44 & -48 & 10 & 36 & 136 & 4.65 \\
\hline Rt & Precentral gyrus & 6 & 28 & -2 & 50 & 91 & 4.65 \\
\hline \multicolumn{8}{|c|}{ Parietal lobe } \\
\hline $\mathrm{Lt}$ & Superior parietal lobule & 7 & -18 & -62 & 64 & 878 & 6.18 \\
\hline $\mathrm{Rt}$ & Superior parietal lobule & 5 & 16 & -52 & 66 & $|, 54|$ & 6.01 \\
\hline $\mathrm{Lt}$ & Superior parietal lobule & 7 & -20 & -74 & 46 & 92 & 5.32 \\
\hline $\mathrm{Lt}$ & Inferior parietal lobule & 2 & -44 & -28 & 40 & 49 & 3.68 \\
\hline \multicolumn{8}{|c|}{ Temporal lobe } \\
\hline Rt & Middle temporal lobe & 37 & 46 & -66 & 6 & 669 & 11.29 \\
\hline $\mathrm{Lt}$ & Middle temporal lobe & 37 & -56 & -68 & 2 & 557 & 6.12 \\
\hline \multicolumn{8}{|c|}{ Occipital lobe } \\
\hline $\mathrm{Lt}$ & Middle occipital lobe & 18 & -26 & -88 & 12 & 31 & 4.16 \\
\hline \multicolumn{8}{|c|}{ B: schizophrenia group } \\
\hline \multicolumn{8}{|c|}{ Frontal lobe } \\
\hline Rt & Middle frontal gyrus & 6 & 28 & 2 & 50 & 333 & 6.20 \\
\hline $\mathrm{Lt}$ & Precentral gyrus & 6 & -40 & 0 & 44 & 153 & 4.49 \\
\hline $\mathrm{Rt}$ & Supplemental motor area & 8 & 4 & 22 & 52 & 23 & 3.77 \\
\hline \multicolumn{8}{|c|}{ Parietal lobe } \\
\hline $\mathrm{Lt}$ & Inferior parietal lobule & 40 & -42 & -50 & 56 & 388 & 4.35 \\
\hline Rt & Superior parietal lobule & I & 20 & -46 & 74 & 12 & 4.02 \\
\hline $\mathrm{Rt}$ & Inferior parietal lobule & 40 & 38 & -38 & 46 & 79 & 3.70 \\
\hline Rt & Postcentral gyrus & 3 & 36 & -26 & 46 & 19 & 3.56 \\
\hline \multicolumn{8}{|c|}{ Temporal lobe } \\
\hline Rt & Middle temporal gyrus & 37 & 48 & -66 & 6 & 1,694 & 8.85 \\
\hline $\mathrm{Rt}$ & Inferior temporal gyrus & 20 & 46 & -22 & -12 & 21 & 4.02 \\
\hline \multicolumn{8}{|c|}{ Occipital lobe } \\
\hline $\mathrm{Lt}$ & Middle occipital gyrus & 19 & -48 & -76 & 2 & 971 & 7.00 \\
\hline Lt & Superior occipital gyrus & 7 & -26 & -70 & 38 & 19 & 3.58 \\
\hline
\end{tabular}

Notes: The clusters showed significant activation for each condition. Each row shows the anatomical location of the activation and the corresponding BA, the MNI coordinates of the peak voxel, and the volume of activation. A one-sample $f$-test at a threshold of $q(F D R)<0.05$ and an extended threshold of ten contiguous voxels was employed. Abbreviations: BA, Brodmann's area; FDR, false discovery rate; Lt, left; MNI, Montreal Neurological Institute; Rt, right; SM, scrambled motion; ST, static condition.

perception, ${ }^{24,33-36}$ we replicated the finding that there is significantly greater activation in bilateral STS during socially and biologically meaningful motion stimuli in normal controls. However, contrary to previous findings, ${ }^{15}$ we also found enhanced activation in bilateral STS in the patient group.

In our current experiment, we adopted a blocked design visual paradigm that induced robust bilateral STS activation to socially relevant motion stimuli in both groups. In a previous study, Kim et al introduced an event-related design and did not find significant differences in STS activation when contrasting BM with SM in schizophrenia patients. ${ }^{15}$ The discrepancy of the results found in the current experiment and this previous neuroimaging study could be due to the difference of the experimental design, as Kim et al used an event-related design, while we employed a blocked design.
Blocked designs typically yield more robust activation patterns and larger changes in BOLD signals due to increased statistical power. ${ }^{37}$ In fact, Kim et al also found significant differences in STS activation in their patients when contrasting $\mathrm{BM}$ with $\mathrm{SM}$ in a blocked design during their functional localization procedure. ${ }^{15}$ Although it is not the case with point-light animation tasks, Thakkar et al showed significant differences in STS activation in a schizophrenia patient group during the animated versus nonanimated action observation in their blocked design experiment. ${ }^{26}$

According to our initial hypothesis, we explored atypical neural activation in other regions of the social brain network when contrasting the BM stimuli (which contained socially and biologically relevant motion stimuli) with SM stimuli (which contained SM) in both groups. To our knowledge, 
Table 4 Significantly activated clusters for the BM-SM contrast

\begin{tabular}{|c|c|c|c|c|c|c|c|}
\hline \multirow[t]{2}{*}{ Region } & & \multirow[t]{2}{*}{ BA } & \multicolumn{3}{|c|}{ MNI coordinates } & \multirow[t]{2}{*}{ Cluster size } & \multirow[t]{2}{*}{$t$-value } \\
\hline & & & $\mathbf{x}$ & $y$ & $\mathbf{z}$ & & \\
\hline \multicolumn{8}{|c|}{ A: normal group ${ }^{a}$} \\
\hline \multicolumn{8}{|c|}{ Frontal lobe } \\
\hline Rt & Medial orbitofrontal cortex & 11 & 2 & 40 & -10 & 190 & 4.52 \\
\hline Lt & Superior frontal gyrus & 9 & -14 & 48 & 36 & 63 & 4.40 \\
\hline $\mathrm{Lt}$ & Medial superior frontal cortex & 10 & -6 & 66 & 10 & 225 & 4.33 \\
\hline Rt & Superior frontal gyrus & 6 & 14 & 14 & 52 & 16 & 3.99 \\
\hline Rt & Precentral gyrus & 6 & 50 & -6 & 48 & 10 & 3.69 \\
\hline Rt & Medial orbitofrontal cortex & 11 & 2 & 40 & -10 & 190 & 4.52 \\
\hline Lt & Superior frontal gyrus & 9 & -14 & 48 & 36 & 63 & 4.40 \\
\hline \multicolumn{8}{|c|}{ Parietal lobe } \\
\hline $\mathrm{Lt}$ & Precuneus & 5 & -4 & -44 & 50 & $\mathrm{I}, 287$ & 5.66 \\
\hline Lt & Precuneus & 23 & -8 & -56 & 24 & 287 & 4.56 \\
\hline Rt & Paracentral lobule & 4 & 0 & -28 & 74 & 38 & 3.83 \\
\hline \multicolumn{8}{|c|}{ Temporal lobe } \\
\hline $\mathrm{Lt}$ & Superior temporal gyrus & 48 & -50 & -24 & 14 & 113 & 5.27 \\
\hline Lt & Superior temporal sulcus & 21 & -54 & -56 & 22 & 275 & 5.04 \\
\hline Rt & Superior temporal sulcus & 21 & 62 & -52 & 18 & 100 & 4.31 \\
\hline Rt & Heschel gyrus & 48 & 52 & -6 & 8 & 18 & 4.02 \\
\hline \multicolumn{8}{|c|}{ Occipital lobe } \\
\hline $\mathrm{Rt}$ & Lingual gyrus & 27 & 8 & -40 & 4 & 10 & 3.75 \\
\hline Lt & Lingual gyrus & -6 & -18 & -60 & -6 & 10 & 3.69 \\
\hline \multicolumn{8}{|c|}{ Cingulate and hippocampus } \\
\hline $\mathrm{Lt}$ & Middle cingulate & 24 & -8 & 12 & 32 & 23 & 4.13 \\
\hline Lt & Hippocampus & 35 & -16 & -10 & -18 & 36 & 3.89 \\
\hline \multicolumn{8}{|c|}{ B: schizophrenia group ${ }^{a}$} \\
\hline \multicolumn{8}{|c|}{ Temporal lobe } \\
\hline Lt & Superior temporal sulcus & 22 & -60 & -44 & 10 & 21 & 6.66 \\
\hline Rt & Superior temporal sulcus & 21 & 62 & -52 & 18 & 18 & 5.57 \\
\hline \multicolumn{8}{|c|}{ C: comparison between groups (normal versus schizophrenia) ${ }^{\mathrm{b}}$} \\
\hline \multicolumn{8}{|c|}{ Frontal lobe } \\
\hline Lt & Superior frontal gyrus & 9 & -14 & 48 & 34 & 29 & 4.20 \\
\hline \multicolumn{8}{|c|}{ Parietal lobe } \\
\hline $\mathrm{Lt}$ & Supramarginal gyrus & 48 & -50 & -24 & 16 & 13 & 4.15 \\
\hline \multicolumn{8}{|c|}{ Occipital lobe } \\
\hline $\mathrm{Lt}$ & Cuneus & 18 & 0 & -84 & 28 & 41 & 4.66 \\
\hline $\mathrm{Lt}$ & Precuneus & 7 & -8 & -72 & 38 & 10 & 3.80 \\
\hline \multicolumn{8}{|c|}{ Cingulate and hippocampus } \\
\hline $\mathrm{Lt}$ & Middle cingulate & & -2 & -44 & 50 & 28 & 4.50 \\
\hline
\end{tabular}

Notes: The clusters showed significant activation for each condition. Each row shows the anatomical location of the activation and the corresponding BA, the MNI coordinates of the peak voxel, and the volume of activation. ${ }^{a} \mathrm{~A}$ one-sample $f$-test at a threshold of $\mathrm{q}(\mathrm{FDR})<0.05$ and an extended threshold of ten contiguous voxels. ${ }^{b} \mathrm{~A}$ two-sample $f$-test at a threshold of $P<0.0005$ (uncorrected) and an extended threshold of ten contiguous voxels.

Abbreviations: BA, Brodmann's area; BM, biological motion; FDR, false discovery rate; Lt, left; MNI, Montreal Neurological Institute; Rt, right; SM, scrambled motion.

this study is the first to report greater activation in regions of the social brain network, specifically left MPFC and left TPJ, in the normal control group. Critically, these regions of the social brain network did not yield significantly different activations to BM versus SM in the patient group (Table 4, Figure 3). As stated in previous literature on the functional role in the TPJ, MPFC, and precuneus, these brain regions are known to be involved in ToM, ${ }^{38-40}$ such as interpreting other's intention and behaviors. Overwalle and Baetens have pointed out that this ToM network was activated by observing moving body parts in two cases such as (1) observing intended action, pretended action, or unexpected motion, and (2) understanding the intent of a social agent at the level of goal or general intention. ${ }^{39}$ Supporting this possibility, some of our BM stimuli used in the current experiment representing pretended actions such as picking up an invisible object, swinging an invisible bat or golf club, standing up from an invisible chair, and sitting down on an invisible chair. We speculate that these 
actions can induce activation in brain regions related to ToM task in our control subjects. On the other hand, patients with schizophrenia failed to demonstrate similar neural patterns of response in these ToM-related brain regions, perhaps due to a lack in the recruitment of neural circuits needed for ToM processing, as others have previously reported. ${ }^{3,41}$ Because we did not collect any behavioral data related to ToM in our subjects, further study is needed to confirm our speculation. The SOFAS score of our subjects did not significantly correlate with the activation of ToM-related area in BM versus SM conditions in our schizophrenia patients $(r=0.07, P=0.80$ in MPFC and $r=-0.25, P=0.33$ in TPJ).

Another possibility for the atypical neural activation patterns we observed in the schizophrenic group during BM versus SM perception could be related to abnormalities in visual processing. ${ }^{42,43}$ We further explored the neural responses to motion in patients with schizophrenia. To confirm the previous findings regarding atypical neural responses to motion in patients with schizophrenia, ${ }^{13,44}$ we contrasted the neural activation induced by BM or SM stimuli with ST stimuli. We found strong neural activation in bilateral temporal lobes, including hMT+ areas, where it still remains unclear whether a functional deficiency exists there in schizophrenia patients. ${ }^{13,15,45}$ Our current finding suggests that neural activation in hMT+ was preserved in our patients.

Furthermore, the SM-ST contrast revealed significant activation in the bilateral SPL for normal control and right SPL for patients in the schizophrenia group. This brain region has been reported to be involved in attentional tracking of moving objects. ${ }^{46}$ In a similar line of research, Thompson et al have shown that increased responses in the bilateral SPL were observed when spatially fragmented moving human figures were presented. ${ }^{47}$ Those authors suggested that subjects would need to track the motion of body parts independently in a manner similar to tracking the motion of independent objects for the absence of a body configuration. ${ }^{47}$ Although the visual stimuli that we used here were different from those in the study by Thompson et al it is possible that activation in the SPL was elicited by the SM stimuli, which also consisted of moving dots without a body configuration. Based on our current results, it seems that the neural activation in SPL was preserved in the schizophrenia patients in our setting.

There are several limitations in our study. First, because we did not collect any ToM-related behavioral data, we are unable to confirm the relationship between the abnormal brain activation in MPFC and TPJ we found in this experiment to ToM deficiency in our subjects. Further study is needed on this point to clarify this potential relationship. Second, all of the patients in our study undertook neuroleptic medications at the time of testing, and the effects of medication cannot be excluded. Furthermore, patients in our study had various durations of illness (0.6-12.3 years) and various symptom severities (the PANSS total score ranged from 35 to 101). Further studies are needed to address the effect of medical treatment and perhaps examine a more homogenous sample of patients (eg, only first-episode schizophrenia patients).

In conclusion, we found differential neural activation induced by socially and biologically relevant motion stimuli in bilateral STS for both groups, but differential activation to these same stimuli in the MPFC and TPJ regions in only normal controls. This finding provides insight into the relationship between visual deficiencies and social cognitive impairment in patients with schizophrenia. Because both visual and social cognitive impairments are well-known and functionally significant abnormalities, an increased understanding of their relationship is etiologically important if we are to better understand schizophrenia and identify biomarkers of this illness. Dysfunction of the social brain network during BM perception in patients with schizophrenia could be a hallmark of their social cognitive deficits. ${ }^{18}$ Further studies are needed to explore the relationship between the impairment of BM perception and social cognitive dysfunctions in schizophrenia.

\section{Acknowledgments}

We thank Dr Matsuda of Iwate Prefectural University for providing the motion-capture data. We thank Fumiko Hoeft, $\mathrm{MD}, \mathrm{PhD}$, and Tiffany $\mathrm{Ho}, \mathrm{PhD}$, for editorial assistance.

\section{Disclosure}

The authors report no conflicts of interest in this work.

\section{References}

1. Green MF, Leitman DI. Social cognition in schizophrenia. Schizophr Bull. 2008;34(4):670-672.

2. Butler PD, Zemon V, Schechter I, et al. Early-stage visual processing and cortical amplification deficits in schizophrenia. Arch Gen Psychiatry. 2005;62(5):495-504.

3. Brunet E, Sarfati Y, Hardy-Baylé MC, Decety J. Abnormalities of brain function during a nonverbal theory of mind task in schizophrenia. Neuropsychologia. 2003;41(12):1574-1582.

4. Brune M. "Theory of mind" in schizophrenia: a review of the literature. Schizophr Bull. 2005;31(1):21-42.

5. Bjorkquist OA, Herbener ES. Social perception in schizophrenia: evidence of temporo-occipital and prefrontal dysfunction. Psychiatry Res. 2013;212(3):175-182.

6. Benedetti F, Bernasconi A, Bosia M, et al. Functional and structural brain correlates of theory of mind and empathy deficits in schizophrenia. Schizophr Res. 2009;114(1-3):154-160. 
7. Butler PD, Silverstein SM, Dakin SC. Visual perception and its impairment in schizophrenia. Biol Psychiatry. 2008;64(1):40-47.

8. Chen Y. Abnormal visual motion processing in schizophrenia: a review of research progress. Schizophr Bull. 2011;37(4):709-715.

9. Martínez A, Hillyard SA, Dias EC, et al. Magnocellular pathway impairment in schizophrenia: evidence from functional magnetic resonance imaging. $J$ Neurosci. 2008;28(30):7492-7500.

10. Butler PD, Schechter I, Zemon V, et al. Dysfunction of earlystage visual processing in schizophrenia. Am J Psychiatry. 2001; 158(7):1126-1133.

11. Schechter I, Butler PD, Silipo G, Zemon V, Javitt DC. Magnocellular and parvocellular contributions to backward masking dysfunction in schizophrenia. Schizophr Res. 2003;64(2-3):91-101.

12. Clementz BA, McDowell JE, Dobkins KR. Compromised speed discrimination among schizophrenia patients when viewing smooth pursuit targets. Schizophr Res. 2007;95(1-3):61-64.

13. Chen Y, Grossman ED, Bidwell LC, et al. Differential activation patterns of occipital and prefrontal cortices during motion processing: evidence from normal and schizophrenic brains. Cogn Affect Behav Neurosci. 2008;8(3):293-303.

14. Kim J, Doop ML, Blake R, Park S. Impaired visual recognition of biological motion in schizophrenia. Schizophr Res. 2005;77(2-3):299-307.

15. Kim J, Park S, Blake R. Perception of biological motion in schizophrenia and healthy individuals: a behavioral and FMRI study. PLoS One. 2011;6(5):e19971.

16. Brittain P, Ffytche DH, McKendrick A, Surguladze S. Visual processing, social cognition and functional outcome in schizophrenia. Psychiatry Res. 2010;178(2):270-275.

17. Kim J, Norton D, McBain R, Ongur D, Chen Y. Deficient biological motion perception in schizophrenia: results from a motion noise paradigm. Front Psychol. 2013;4:391.

18. Pavlova MA. Biological motion processing as a hallmark of social cognition. Cereb Cortex. 2012;22(5):981-995.

19. Johansson G. Visual perception of bending motion and a model for its analysis. Percept Psychophys. 1973;14(2):201-211.

20. Dittrich WH. Action categories and the perception of biological motion. Perception. 1993;22(1):15-22.

21. Dittrich WH, Troscianko T, Lea SE, Morgan D. Perception of emotion from dynamic point-light displays represented in dance. Perception. 1996;25(6):727-738.

22. Troje NF. Decomposing biological motion: a framework for analysis and synthesis of human gait patterns. $J$ Vis. 2002;2(5):371-387.

23. Saygin AP. Superior temporal and premotor brain areas necessary for biological motion perception. Brain. 2007;130(Pt 9):2452-2461.

24. Bonda E, Petrides M, Ostry D, Evans A. Specific involvement of human parietal systems and the amygdala in the perception of biological motion. J Neurosci. 1996;16(11):3737-3744.

25. Jastorff J, Orban GA. Human functional magnetic resonance imaging reveals separation and integration of shape and motion cues in biological motion processing. $J$ Neurosci. 2009;29(22):7315-7329.

26. Thakkar KN, Peterman JS, Park S. Altered brain activation during action imitation and observation in schizophrenia: a translational approach to investigating social dysfunction in schizophrenia. Am J Psychiatry. 2014;171(5):539-548.

27. Das P, Lagopoulos J, Coulston CM, Henderson AF, Malhi GS. Mentalizing impairment in schizophrenia: a functional MRI study. Schizophr Res. 2012;134(2-3):158-164.
28. American Psychiatric Association. Diagnostic and Statistical Manual of Mental Disorders, 4th ed. (text rev.). Washington, DC: American Psychiatric Press; 2000.

29. Hirai M, Kakigi R. Differential cortical processing of local and global motion information in biological motion: an event-related potential study. J Vis. 2008;8(16):2.1-217.

30. Grossman ED, Blake R. Brain Areas Active during Visual Perception of Biological Motion. Neuron. 2002;35(6):1167-1175.

31. Hirai M, Fukushima H, Hiraki K. An event-related potentials study of biological motion perception in humans. Neurosci Lett. 2003;344(1):41-44.

32. Jokisch D, Troje NF, Koch B, Schwarz M, Daum I. Differential involvement of the cerebellum in biological and coherent motion perception. Eur J Neurosci. 2005;21(12):3439-3446.

33. Grossman ED, Donnelly M, Price R, et al. Brain areas involved in perception of biological motion. J Cogn Neurosci. 2000;12(5): 711-720.

34. Grèzes J, Decety J. Functional anatomy of execution, mental simulation, observation, and verb generation of actions: a meta-analysis. Hum Brain Mapp. 2001;12(1):1-19.

35. Peuskens H, Vanrie J, Verfaillie K, Orban GA. Specificity of regions processing biological motion. Eur J Neurosci. 2005; 21(10):2864-2875

36. Grossman ED, Jardine NL, Pyles JA. fMR-Adaptation Reveals Invariant Coding of Biological Motion on the Human STS. Front Hum Neurosci. 2010;4:15.

37. Amaro E Jr, Barker GJ. Study design in fMRI: basic principles. Brain Cogn. 2006;60(3):220-232.

38. Frith $\mathrm{CD}$, Frith U. Interacting minds - a biological basis. Science. 1999;286(5445):1692-1695.

39. Amodio DM, Frith CD. Meeting of minds: the medial frontal cortex and social cognition. Nat Rev Neurosci. 2006;7(4):268-277.

40. Van Overwalle F, Baetens K. Understanding others' actions and goals by mirror and mentalizing systems: a meta-analysis. Neuroimage. 2009;48(3):564-584.

41. Singh F, Pineda J, Cadenhead KS. Association of impaired EEG mu wave suppression, negative symptoms and social functioning in biological motion processing in first episode of psychosis. Schizophr Res. 2011;130(s1-s3):182-186.

42. Sergi MJ, Green MF. Social perception and early visual processing in schizophrenia. Schizophr Res. 2003;59(2-3):233-241.

43. Kelemen O, Erdélyi R, Pataki I, Benedek G, Janka Z, Kéri S. Theory of mind and motion perception in schizophrenia. Neuropsychology. 2005;19(4):494-500.

44. Lencer R, Nagel M, Sprenger A, Heide W, Binkofski F. Reduced neuronal activity in the V5 complex underlies smooth-pursuit deficit in schizophrenia: evidence from an fMRI study. Neuroimage. 2005;24(4):1256-1259.

45. Kim D, Wylie G, Pasternak R, Butler PD, Javitt DC. Magnocellular contributions to impaired motion processing in schizophrenia. Schizophr Res. 2006;82(1):1-8.

46. Culham JC, Brandt SA, Cavanagh P, Kanwisher NG, Dale AM, Tootell RB. Cortical fMRI activation produced by attentive tracking of moving targets. J Neurophysiol. 1998;80(5):2657-2670.

47. Thompson JC, Clarke M, Stewart T, Puce A. Configural processing of biological motion in human superior temporal sulcus. $J$ Neurosci. 2005;25(39):9059-9066.

Neuropsychiatric Disease and Treatment

\section{Publish your work in this journal}

Neuropsychiatric Disease and Treatment is an international, peerreviewed journal of clinical therapeutics and pharmacology focusing on concise rapid reporting of clinical or pre-clinical studies on a range of neuropsychiatric and neurological disorders. This journal is indexed on PubMed Central, the 'PsycINFO' database and CAS,

\section{Dovepress}

and is the official journal of The International Neuropsychiatric Association (INA). The manuscript management system is completely online and includes a very quick and fair peer-review system, which is all easy to use. Visit http://www.dovepress.com/testimonials.php to read real quotes from published authors. 